\title{
Impact of Dedicated Brain PET on Intended Patient Management in Participants of the National Oncologic PET Registry
}

\author{
Bruce E. Hillner, ${ }^{1,2}$ Barry A. Siegel, ${ }^{3,4}$ Anthony F. Shields, ${ }^{5}$ Fenghai Duan, ${ }^{6}$ \\ Ilana F. Gareen, ${ }^{6}$ Lucy Hanna, ${ }^{6}$ R. Edward Coleman ${ }^{7}$ \\ ${ }^{1}$ Department of Internal Medicine, Virginia Commonwealth University, 1101 E. Marshall St. Room 7013, Richmond, VA 23298-0170, USA \\ ${ }^{2}$ Massey Cancer Center, Virginia Commonwealth University, Richmond, VA, USA \\ ${ }^{3}$ Division of Nuclear Medicine, Mallinckrodt Institute of Radiology, Washington University School of Medicine, St. Louis, MO, USA \\ ${ }^{4}$ Siteman Cancer Center, Washington University School of Medicine, St. Louis, MO, USA \\ ${ }^{5}$ Karmanos Cancer Institute, Wayne State University, Detroit, MI, USA \\ ${ }^{6}$ Center for Statistical Sciences, Brown University, Providence, RI, USA \\ ${ }^{7}$ Department of Radiology, Duke University School of Medicine, Durham, NC, USA
}

\begin{abstract}
Purpose: This study seeks to assess the impact of dedicated brain positron emission tomography (PET) with 2-deoxy-2-[ $\left[{ }^{18} \mathrm{~F}\right] \mathrm{fluoro}-\mathrm{D}-\mathrm{glucose}$ on intended management of patients with primary and metastatic brain tumors.

Procedures: We analyzed demographic characteristics and evaluated change in intended management after PET, using previously described metrics, for patients in the National Oncologic PET Registry (NOPR) undergoing dedicated brain PET. For cases of primary brain tumors, comparisons to the overall NOPR cohort were made.

Patient Profile: Between December 2006 and April 2009, 509 dedicated brain PET scans were done on 479 patients-367 $(72.1 \%)$ for suspected or proven primary brain tumors and 142 $(27.9 \%)$ for brain metastases. Compared with the overall NOPR cohort, subjects in the dedicated brain cohort were younger ( $41.3 \%$ less than 65 years vs. $10.5 \%$ overall, $p<0.0001$ ) and more frequently had functional limitations from their cancers $(78.6 \%$ vs. $62.3 \%$ overall; odds ratio (OR) 2.2, 95\% Cl 1.8-2.8).

Results: The pre-PET patient management plans in the primary brain tumor and metastasis subgroups were similar. A pre-PET plan of tissue biopsy was slightly more frequent than one of the treatments $(31.3 \%$ vs. $28.6 \%)$ in the primary brain tumor subgroup and was more common than in the overall NOPR cohort (14.2\%; OR 2.8, 95\% Cl 2.2-3.5). Changes from treatment to non-treatment also were more frequent than in the overall NOPR cohort $(13.4 \%$ vs. $7.7 \%$; OR 1.9, 95\% Cl 1.3-2.5).

Conclusions: Among NOPR patients, dedicated brain PET was associated with similar net changes in intended management as in the overall NOPR cohort. However, brain PET patients were younger, more likely to be symptomatic, and less likely to have a change in management from non-treatment to treatment as a post-PET plan.
\end{abstract}

Key words: Positron emission tomography, Brain neoplasms, Registry, Prospective studies 


\section{Introduction}

$\mathrm{T}$ he role of 2-deoxy-2- $\left[{ }^{18} \mathrm{~F}\right]$ fluoro-D-glucose positron emission tomography (FDG-PET) in the management of malignant brain tumors is uncertain [1]. Advocates of PET suggest that it has several roles complementary to those of magnetic resonance imaging (MRI) and computed tomography (CT) in the evaluation of patients with primary or metastatic tumors of the brain $[2,3]$. These complementary roles reflect PET's unique ability to assess tissue glucose utilization, which facilitates evaluation of tumor grade, identification of optimal sites for stereotactic biopsy and determination of prognosis during the initial evaluation of patients with brain tumors. As a tool for evaluation following treatment, PET can also be useful for detecting residual tumor after surgery, distinguishing between radiation necrosis and recurrent tumor [4], and monitoring tumor progression. Additionally, in patients with presumed metastatic lesions to the brain seen on MRI, whole-body PET may be useful in determining the origin of unidentified primary tumors [1].

Since 2006, the National Oncologic PET Registry (NOPR) has collected data on Medicare beneficiaries with known or suspected cancers undergoing PET for a wide variety of uncommon cancer types, including brain cancers, under Centers of Medicare and Medicaid Services policy called "coverage with evidence development" [5-7]. We have previously reported findings from NOPR on the impact of whole-body PET on intended management in patients with proven or suspected cancer. We found that the effect of PET on changing intended management was highly consistent across cancer types and indications [8,9].

The objective of this study is to assess the impact of dedicated brain PET on intended patient management in patients with primary and metastatic brain tumors based on data in the NOPR.

\section{Patients and Methods}

The NOPR is a prospective data registry that collects information from the PET facility, the physician requesting the PET scan, and the interpreting physician's PET report. Detailed descriptions of NOPR operations, human subject protection procedures, and results for the impact of PET on physicians' intended management were reported previously [8-10].

In brief, the PET facility is responsible for collecting information from the referring physician on a pre-PET form and a post-PET form. The pre-PET form focuses on information regarding the specific indication for testing, the patient's cancer type (if known) and working stage assessment, the patient's performance status, whether the referring physician will also be the treating physician, and the referring physician's management plan if PET was not available. Once the PET scan has been completed, the PET facility uploads the PET report to the database. The final step is the completion of the post-PET form by the referring physician to assess the management plan in light of the PET findings.
The primary endpoint was the impact of PET on physicians' intended management dichotomized as either treatment (e.g., surgery, chemotherapy, radiation, or other treatment, alone or in combination) or non-treatment (e.g., observation, alternative imaging or other noninvasive testing, biopsy, or supportive care). A change in management was defined as a switch from treatment to non-treatment or vice versa.

NOPR began enrolling patients in May 2006. In December 2006, the data collection procedures were changed to stratify the PET imaging procedures into three categories: body PET (or PET/ CT), dedicated brain PET only, or both body PET (or PET/CT) and dedicated brain PET. This analysis uses only cases of dedicated brain PET (CPT® code 78608 ) because where both body and dedicated brain imaging were performed, the referring physician's responses did not discriminate the impact of each component on intended management. The cohort does not include cases where a body PET or PET/CT acquisition (CPT ${ }^{\circledR}$ codes 78811-78816) included the entire brain. In addition, cases where PET was requested for monitoring of ongoing treatment were excluded.

\section{Statistical Analysis}

A change in intended management after PET was modeled as binary variable on the basis of a binomial distribution. Changes were defined at the PET scan level and were assumed to be independent for different patients or indication for testing.

For the comparison of the change of intended management in the dedicated brain PET cohort with that in the overall NOPR cohort (excluding treatment monitoring), we used chi-square analysis. For these comparisons, we restricted our study population to those patients with primary brain tumors, excluding patients with proven or suspected brain metastases because of the small number of the latter. Odds ratios (ORs) were calculated to quantify the association between two binary variables. The $95 \%$ confidence intervals are constructed with the exact method for binomial distribution. All statistical analyses were carried out using SAS version 9.1 (SAS Institute, Cary, NC).

\section{Results}

This report includes data from 509 dedicated brain PET scans performed between December 1, 2006 and April 3, 2009 on 479 patients. During the same interval, additional four studies involving both body PET (or PET/CT) and dedicated brain PET were performed in two patients. The entire NOPR cohort for this period consisted of 74,932 scans in 62,122 patients.

The clinical profile of the cohort is summarized in Table 1. The referring physicians recorded the cancer type as primary brain tumor $(n=367)$ and brain metastases $(n=142)$, including suspected or proven brain metastases of unknown primary origin $(n=28)$. The indication for PET imaging in patients with primary brain tumors was diagnosis in $21.2 \%$, initial staging in $4.1 \%$, restaging in $16.6 \%$, and detection of suspected recurrence in $58.0 \%$. Thirty-seven cases, where the pre-PET form indicated that the patient had a primary brain tumor, were reclassified as suspected or proven brain metastases since the summary stage on the pre-PET form stated that the patient already had known non-brain metastatic sites. Eighty percent of the scans were performed with PET/CT scanners. 
Compared with the overall NOPR cohort, subjects in the brain-only cohort were younger $(41.3 \%$ less than 65 years vs. $10.5 \%$ overall, $p<0.0001)$ and more often symptomatic from their cancers [ECOG] performance score $\geq 1$ in $78.6 \%$ vs. $62.3 \%$ overall (OR $2.2,95 \%$ CI $1.8-2.8$ ) or $\geq 2$ in $25.5 \%$ vs. $12.6 \%$ overall (OR 2.4, 95\% CI 1.9-2.9).

The pre-PET management plans in the primary brain tumor and metastasis subgroups were similar. The most frequent plan of intended management before PET, assuming PET was not available, was alternative imaging. For the primary tumor tumor-only subgroup, the pre-PET plan was slightly more frequently tissue biopsy than treatment $(31.3 \%$ vs. $28.6 \%$ ); this frequency of planned biopsy if PET was not available was more common than in the overall NOPR cohort (14.2\%; OR 2.8, 95\% CI 2.2-3.5).

\section{Outcome Results}

Table 2 summarizes the changes in intended management when the pre- and post-PET plans were classified as treatment vs. non-treatment. The frequency of change in intended management associated with PET was $38.2 \%$ in the primary brain tumor subgroup and $35.2 \%$ in the brain metastasis subgroup. The frequency of change in the primary brain tumor subgroup was similar to that in the overall NOPR cohort $(37.0 \%$; OR $=1.1,95 \%$ CI $0.8-1.3)$.

In the primary brain tumor subgroup, there were modest differences when comparing scans done for diagnosis or initial staging ( $n=93)$ vs. restaging or detection of suspected recurrence $(n=274)$. Any form of treatment as the post-PET plan was slightly more common in restaging/suspected recurrence cases $(41.7 \%)$ than in diagnosis/initial staging cases $(35.5 \%)$. Overall, changes from treatment to nontreatment were more frequent in the primary brain tumor subgroup than in the overall NOPR cohort (13.4\% vs. 7.7\%; OR $1.9,95 \%$ CI $1.3-2.5$ ).

Table 3 summarizes the changes in intended management when stratified by specific intended actions for the two brain tumor subgroups. For both subgroups, the two most frequent pre-PET strategies - tissue biopsy or alternative imaging - involved additional data gathering. PostPET, the intended management was revised in more than

Table 1. Profile of dedicated brain PET studies in NOPR

\begin{tabular}{|c|c|c|c|c|}
\hline & \multicolumn{2}{|c|}{ Primary brain tumors } & \multicolumn{2}{|c|}{ Proven or suspected brain metastasis } \\
\hline & Number & $\%$ & Number & $\%$ \\
\hline Total scans (Total patients) & $367(343)$ & & $142(139)$ & \\
\hline Age, mean (years) & 61.8 & & 67.9 & \\
\hline Median & 66 & & 70 & \\
\hline Range & $25-97$ & & $29-88$ & \\
\hline \multicolumn{5}{|l|}{ Primary site of metastases to brain } \\
\hline Unknown primary & $\mathrm{NA}^{\mathrm{a}}$ & & 28 & 26.7 \\
\hline Lung, small cell & NA & & 14 & 13.3 \\
\hline Prostate & NA & & 12 & 11.4 \\
\hline Kidney and other urinary tract & NA & & 9 & 8.6 \\
\hline Ovary and uterine adnexa & NA & & 6 & 5.7 \\
\hline Other & NA & & 36 & 34. \\
\hline \multicolumn{5}{|l|}{ Performance status (ECOG scale) } \\
\hline Asymptomatic: fully active (0) & 76 & 20.7 & 33 & 23.2 \\
\hline Symptomatic, fully ambulatory (1) & 196 & 53.4 & 74 & 52.1 \\
\hline Symptomatic in bed $<50 \%$ of the day (2) & 60 & 16.3 & 27 & 19.0 \\
\hline Symptomatic in bed $>50 \%$ of the day (3) & 28 & 7.6 & 7 & 4.9 \\
\hline Bedridden (4) & 7 & 1.9 & 1 & 0.7 \\
\hline \multicolumn{5}{|l|}{ Indication for PET } \\
\hline Diagnosis & 78 & 21.3 & a & a \\
\hline Initial Staging & 15 & 4.1 & a & a \\
\hline Restaging & 61 & 16.6 & a & a \\
\hline Suspected Recurrences & 213 & 58.0 & a & a \\
\hline \multicolumn{5}{|l|}{ Pre-PET stage } \\
\hline No evidence of disease/remission & 17 & 4.6 & 3 & 2.1 \\
\hline Localized only & 218 & 59.4 & 18 & 12.6 \\
\hline Regional & 24 & 6.5 & 6 & 4.2 \\
\hline Single site of metastasis & NA & & 45 & 31.6 \\
\hline Multiple sites of metastases & NA & & 40 & 28.1 \\
\hline Unknown or uncertain & 108 & 29.4 & 30 & 21.1 \\
\hline \multicolumn{5}{|l|}{ Pre-PET intended management } \\
\hline Observation & 25 & 6.8 & 17 & 12.0 \\
\hline Additional Imaging & 122 & 33.3 & 54 & 38.0 \\
\hline Tissue Biopsy & 115 & 31.3 & 33 & 23.2 \\
\hline Treatment & 105 & 28.6 & 38 & 26.8 \\
\hline Curative & 48 & 45.7 & 18 & 47.4 \\
\hline Palliative & 57 & 54.3 & 20 & 52.6 \\
\hline
\end{tabular}

$N A$ not applicable, ECOG Eastern Cooperative Oncology Group

${ }^{a}$ Not calculated because of reclassification of 37 cases (see text) 
Table 2. Change in intended management

\begin{tabular}{|c|c|c|c|c|c|c|c|}
\hline \multirow[t]{4}{*}{ Pre-PET } & \multirow[t]{4}{*}{ Post-PET } & \multirow{2}{*}{\multicolumn{2}{|c|}{$\begin{array}{l}\text { Diagnosis or initial staging } \\
\text { Primary brain tumors }\end{array}$}} & \multicolumn{2}{|c|}{ Restaging or suspected recurrence } & \multicolumn{2}{|c|}{ Known or suspected brain metastasis } \\
\hline & & & & Primary $b$ & & & \\
\hline & & \multicolumn{2}{|l|}{$(n=93)$} & \multicolumn{2}{|l|}{$(n=274)$} & \multicolumn{2}{|l|}{$(n=142)$} \\
\hline & & Number & $\%$ & Number & $\%$ & Number & $\%$ \\
\hline Non-treatment & Non-treatment & 48 & 51.6 & 123 & 44.9 & 68 & 47.9 \\
\hline Treatment & Treatment & 11 & 11.8 & 45 & 16.4 & 24 & 16.9 \\
\hline Non-treatment & Treatment & 22 & 23.7 & 69 & 25.2 & 36 & 25.4 \\
\hline Treatment & Non-treatment & 12 & 12.9 & 37 & 13.5 & 14 & 9.9 \\
\hline
\end{tabular}

$80 \%$ of cases to either observation or treatment. These revised post-PET plans were about equally divided between observation and treatment.

For the primary brain tumor subgroup, a change in the pre-PET plan from treatment to non-treatment was more common $(46.7 \%)$ than the converse $(34.7 \%)$. Of the primary brain tumor patients with a pre-PET plan of treatment, the post-PET plan had a treatment goal change in $28.6 \%$.

\section{Discussion}

Dedicated brain PET for the evaluation of suspected or proven primary brain tumor or brain metastasis represents a narrow spectrum of the potential uses of PET in oncology. In part, this reflects the limited sensitivity of FDG imaging for detection of brain tumors, particularly those with low to moderate levels of FDG uptake, against a background of high FDG uptake in normal gray matter [2]. MRI, including newer perfusion and diffusion methods, has higher sensitivity and is the dominant modality for brain tumor imaging in current practice [11]. Nonetheless, PET provides complementary information on cancer metabolism that is correlated with tumor grade and prognosis at diagnosis and aids in detecting persistent or recurrent tumor after therapy.

In this report, we describe the experience with oncologic brain PET among patients participating in the NOPR. This report represents the largest series, to our knowledge, addressing the use and impact of dedicated brain PET in patients with brain tumors. Our data indicate that dedicated brain PET is used relatively uncommonly for oncologic indications, accounting for only $0.67 \%$ of NOPR PET scans. While only Medicare beneficiaries were eligible for inclusion in this registry, over $40 \%$ of the patients were less than 65 years of age, the usual threshold for Medicare eligibility.
This is likely because younger participants with brain cancer already had qualified for disability (and thus for Medicare coverage) as a consequence of their disease. That younger patients had to demonstrate disability to qualify for Medicare coverage may explain the higher rates of functional impairment (inferred from ECOG performance scores) observed in the dedicated brain PET cohort as opposed to the NOPR population overall ( $79.3 \%$ vs. $64.3 \%$, respectively).

We found that the frequency of change in intended management associated with dedicated brain PET was similar to that found overall for the aggregate of other cancers in NOPR, yet the changes in intended management as a consequence of PET were less frequently associated with the initiation of therapy in subjects with brain tumors. Changes in intended management from treatment to nontreatment were more frequent than the converse. Among patients with a pre-PET plan of tissue biopsy, less than $16 \%$ continued to have biopsy as the intended management after PET. The avoidance of brain biopsy could result in significant cost savings, but further tracking of Medicare claims is needed to evaluate if such savings are realized.

A major limitation of the NOPR is the inability to determine whether the intended changes in management confer a benefit in long-term outcomes. Furthermore, the registry cannot document that referring physicians actually completed the planned management changes. A data linkage to the Medicare claims of NOPR participants is in process to address this important question.

Although brain metastases outnumber primary neoplasms by at least ten to one [12], the use of dedicated brain PET in NOPR to evaluate suspected metastases was infrequent and less common than for primary brain tumors. This suggests that referring physicians were highly selective in their use of dedicated brain PET among patients with metastatic cancer,

Table 3. Impact on intended management strategies associated with dedicated brain PET in NOPR cohort

\begin{tabular}{lllll}
\hline Change from pre-PET to post-PET plan & \multicolumn{2}{l}{ Primary brain tumors $(n=367)$} & & \multicolumn{2}{l}{ Known or suspected brain metastasis $(n=105)$} \\
& Number & $\%$ & & Number \\
\hline Change from tissue biopsy to an alternative strategy & $98 / 115$ & 85.2 & & $27 / 33$ \\
Change from additional imaging to an alternative strategy & $105 / 122$ & 86.1 & $48 / 54$ & 81.8 \\
Change from treatment to non-treatment & $49 / 105$ & 46.7 & $14 / 38$ & 88.9 \\
Change from non-treatment to treatment & $91 / 262$ & 34.7 & $36 / 104$ & 36.8 \\
Change in pre-PET treatment goal & $16 / 56$ & 28.6 & $5 / 24$ & 20.6 \\
\hline
\end{tabular}


which in turn may reflect the known relatively poor sensitivity of FDG-PET for detection of brain metastases $[13,14]$. Still, in general the direction and magnitude of impact of PET on management was similar between groups.

In summary, dedicated brain PET is used very selectively among participants of NOPR. The estimated national incidence of primary brain cancers in the elderly (age over 65 years) is about 3,600 per year [15]. The NOPR data reported herein represents about $4 \%$ of annual incidence. The NOPR patients are younger more often functionally impaired from their disease and rarely undergo a tissue biopsy after PET.

Acknowledgments. Funding for development of the National Oncologic PET Registry was provided by the Academy for Molecular Imaging; but the registry is otherwise self-supported by the fees paid by participating PET facilities.

Conflict of Interest Disclosure. The authors disclose the following potential conflicts of interest: Drs. Coleman, Shields, and Siegel are members of the Medical Advisory Board and stockholders of Radiology Corporation of America, Inc. Drs. Siegel and Shields are members of the Senior Scientific Advisory Board of Siemens Molecular Imaging. Dr Siegel serves on the Comparative Effectiveness Research Advisory Board of GE Healthcare. Dr. Coleman has research funding from GE Healthcare.

Open Access. This article is distributed under the terms of the Creative Commons Attribution Noncommercial License which permits any noncommercial use, distribution, and reproduction in any medium, provided the original author(s) and source are credited.

\section{References}

1. NCCN clinical practice guidelines in oncology. Central nervous system cancers. V.1.2010., 2010. (Accessed at http://www.ncen.org/professionals/ physician gls/PDF/cns.pdf. Accessed June 1, 2010)

2. Fischman AJ (2008) PET imaging of brain tumors. Cancer Treat Res 143:67-92
3. Chen W (2007) Clinical applications of PET in brain tumors. J Nucl Med 48:1468-1481

4. Hustinx R, Pourdehnad M, Kaschten B, Alavi A (2005) PET imaging for differentiating recurrent brain tumor from radiation necrosis. Radiol Clin North Am 43:35-47

5. Centers for Medicare \& Medicaid Services. Pub 100-03 Medicare National Coverage Determinations. Transmittal 31. 2005 http://www. cms.hhs.gov/Transmittals/downloads/R31NCD.pdf

6. Tunis S, Whicher D (2009) The National Oncologic PET Registry: lessons learned for coverage with evidence development. J Am Coll Radiol 6:360-365

7. Tunis SR, Pearson SD (2006) Coverage options for promising technologies: Medicare's 'coverage with evidence development'. Health Aff 25:1218-1230

8. Hillner BE, Siegel BA, Liu D et al (2008) Impact of positron emission tomography/computed tomography and positron emission tomography (PET) alone on expected management of patients with cancer: initial results from the National Oncologic PET Registry. J Clin Oncol 26:2155-2161

9. Hillner BE, Siegel BA, Shields AF et al (2008) Relationship between cancer type and impact of PET and PET/CT on intended management: findings of the National Oncologic PET Registry. J Nucl Med 49:19281935

10. Hillner BE, Liu D, Coleman RE et al (2007) The National Oncologic PET Registry (NOPR): design and analysis plan. J Nucl Med 48:19011908

11. Cha S (2009) Neuroimaging in neuro-oncology. Neurotherapeutics 6:465-477

12. Physician Data Query. National Cancer Institute. Adult Brain Tumors Treatment (Accessed at http://www.cancer.gov/cancertopics/pdq/ treatment/adultbrain/HealthProfessional on July 9, 2010)

13. Griffeth LK, Rich KM, Dehdashti F et al (1993) Brain metastases from non-central nervous system tumors: evaluation with PET. Radiology $186: 37-44$

14. Rohren EM, Provenzale JM, Barboriak DP, Coleman RE (2003) Screening for cerebral metastases with FDG PET in patients undergoing whole-body staging of non-central nervous system malignancy. Radiology 226:181-187

15. National Program of Cancer Registries (NPCR), Centers for Disease Control and Prevention, Department of Health and Human Services. United States Cancer Statistics (USCS): 1999-2006 Cancer Incidence and Mortality Data. Available at: www.cdc.gov/uscs. Accessed August 11,2010 